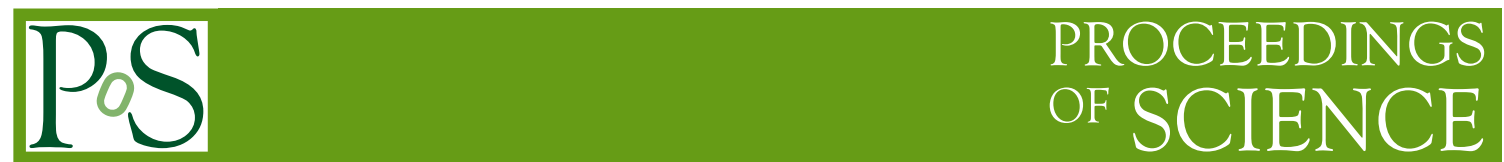

\title{
Recent $\mathrm{LHCb}$ results on pentaquark candidates
}

\author{
Jinlin $\mathbf{F u}^{a, *}$ \\ ${ }^{a}$ University of Chinese Academy of Sciences, Beijing, China \\ E-mail: j.fu@cern.ch
}

The recent $\mathrm{LHCb}$ results related to searches for pentaquark candidates are reported. There are evidences for two new pentaquark candidates $P_{c}(4337)^{+}$and $P_{C S}(4459)^{0}$ obtained from amplitude analysis of $B_{s}^{0} \rightarrow J / \psi p \bar{p}$ and $\Xi_{b}^{-} \rightarrow J / \psi \Lambda K^{-}$decays, respectively.

*** The European Physical Society Conference on High Energy Physics (EPS-HEP2021), *** *** 26-30 July $2021 * * *$

*** Online conference, jointly organized by Universität Hamburg and the research center DESY ***

\footnotetext{
${ }^{*}$ Speaker, on hehalf of the LHCb Collaboration
} 


\section{Introduction}

Beyond the naive quark model, the exotic hadrons, such as pentaquarks containing four quarks and one antiquark, are expected from the theory of QCD. The observation of three pentaquark candidates at LHCb [1], $P_{c}(4312)^{+}, P_{c}(4440)^{+}$, and $P_{c}(4457)^{+}$, indicates the interesting physics related to the thresholds of the charmed mesons and baryons.

The evidences for new structure $P_{c}(4337)^{+}$in $B_{s}^{0} \rightarrow J / \psi p \bar{p}$ decays and $P_{c s}(4459)^{0}$ in $\Xi_{b}^{-} \rightarrow J / \psi \Lambda K^{-}$decays found at $\mathrm{LHCb}$ are present.

\section{Evidence for $P_{c}(4337)^{+}$in $B_{s}^{0} \rightarrow J / \psi p \bar{p}$ decays}

The $B_{s}^{0} \rightarrow J / \psi p \bar{p}$ decays are first observed using $5.2 \mathrm{fb}^{-1}$ data collected by the $\mathrm{LHCb}$ experiment [2]. The measured branching fraction of $B_{s}^{0} \rightarrow J / \psi p \bar{p}$ decays, much higher than the expectation without resonant contributions, indicates the possible pentaquark candidates in $J / \psi p$ system, and glueball or enhancement in $p \bar{p}$ system. The $J / \psi p$ mass distribution in the range [4040,4440] MeV is sensitive to the search for $P_{c}(4312)^{+}$, while the $p \bar{p}$ mass distribution for the possible glueball candidate $f_{J}(2220)$. The thresholds of $\Lambda_{c}^{+} \bar{D}^{0}$ and $\Lambda_{c}^{+} \bar{D}^{* 0}$, around which possible structures might be, are also covered in $B_{s}^{0} \rightarrow J / \psi p \bar{p}$ decays.

An amplitude analysis of flavour-untagged $B_{s}^{0} \rightarrow J / \psi p \bar{p}$ decays is performed using $9 \mathrm{fb}^{-1}$ data collected at $\mathrm{LHCb}$ [3]. About 800 signals are reconstructed with purity of $85 \%$. There are hints of horizontal and vertical bands in the region around (18.8-19.0) $\mathrm{GeV}^{2}$ as present in the $m^{2}(J / \psi p)$ and $m^{2}(J / \psi \bar{p})$ distributions, respectively, as shown in Dalitz plot in Figure 1.

The amplitude model is constructed using helicity formalism under the assumption of $\mathrm{CP}$ symmetry conservation. Three interfering decay chains are considered in the amplitude model: $B_{s}^{0} \rightarrow J / \psi X(\rightarrow p \bar{p}), B_{s}^{0} \rightarrow P_{c}^{+}(\rightarrow J / \psi p) \bar{p}$, and $B_{s}^{0} \rightarrow P_{c}^{-}(\rightarrow J / \psi \bar{p}) p$, as $J / \psi$ is reconstructed by $\mu^{+} \mu^{-}$pair. Since there is no flavour identification for $B_{s}^{0}$ or $\bar{B}_{s}^{0}$, the amplitude analysis is sensitive to the sum of the possible contributions from $P_{c}^{+}$and $P_{c}^{-}$states and not sensitive to different couplings for the $P_{c}^{+}$and $P_{c}^{-}$states.

The baseline model includes a nonresonant (NR) contribution $X \rightarrow p \bar{p}$ with spin-parity quantum number equal to $J^{P}=1^{-}$, which has $S$ waves in both $X$ 's production and decay. The higher values of orbital momentum for $X$ contributions are suppressed due to low $Q$-value in the decay. Models including different NR contributions with other quantum numbers are excluded as a result of significantly worse values of $-2 \log \mathcal{L}$ with respect to the $J^{P}=1^{-}$hypothesis. The fit results of the baseline model are in blue as shown in Figure 2. The baseline model does not describe data well, as discrepancies found in $\cos \theta_{p}, m(J / \psi p)$ and $m(J / \psi \bar{p})$ distributions.

In addition to the contributions in the baseline mode, the default model includes two contributions $P_{c}^{+} \rightarrow J / \psi p$ and $P_{c}^{-} \rightarrow J / \psi \bar{p}$ with the same masses, widths and couplings. The projections of angular and mass distributions are improved as shown in Figure 2. Different $J^{P}$ hypotheses for $P_{c}$ are tested and the signal significance in the range of 3.1 to $3.7 \sigma$ provides the evidence for a new pentaquark candidate. Due to the limited sample size, none of the $J^{P}$ hypotheses can be excluded at 95\% confidence level using the $\mathrm{CL}_{s}$ method. The mass and width of this new pentaquark candidate $P_{c}(4337)^{+}$are measured to be $M_{P_{c}}=4337_{-4-2}^{+7+2} \mathrm{MeV}$ and $\Gamma_{P_{c}}=29_{-12-14}^{+26+14} \mathrm{MeV}$, respectively. There 


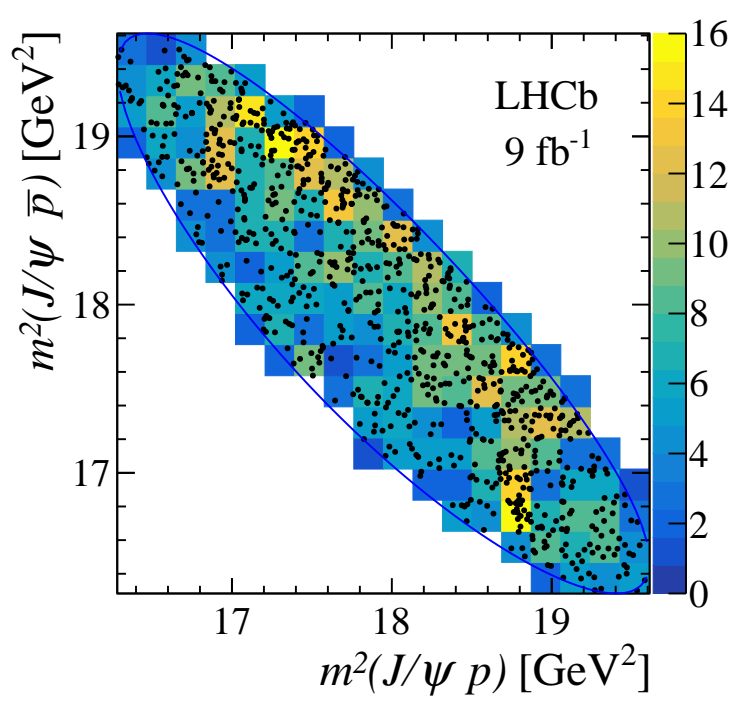

Figure 1: Dalitz distribution for reconstructed candidates (black dots) within the $B_{s}^{0}$ signal region. The colour scale represents the number of candidates in each Dalitz plot interval.

is no evidence for either $P_{c}(4313)^{+}$, or the glueball state $f_{J}(2220)$, or threshold enhancement in $p \bar{p}$ system.

\section{Evidence for $P_{c s}(4459)^{0}$ in $\Xi_{b}^{-} \rightarrow J / \psi \Lambda K^{-}$decays}

The $\Xi_{b}^{-} \rightarrow J / \psi \Lambda K^{-}$decays are first observed using $3 \mathrm{fb}^{-1}$ data collected by the LHCb experiment [4]. The strange counterparts of $P_{c}^{+} \mathrm{s}$, denoted as $P_{c s}^{0}$, are predicted in several papers and has been suggested to search for in $\Xi_{b}^{-} \rightarrow J / \psi \Lambda K^{-}$decays. The excited $\Xi^{-}$spectrum in a mass range of $[1.61,2.70] \mathrm{GeV}$, can be studied in $\Xi_{b}^{-} \rightarrow J / \psi \Lambda K^{-}$decays.

An amplitude analysis of the $\Xi_{b}^{-} \rightarrow J / \psi \Lambda K^{-}$decays is performed using $9 \mathrm{fb}^{-1}$ data collected at LHCb [5]. About 1750 signals are reconstructed with purity of $80 \%$. The $\Xi(1690)^{-}$and $\Xi(1820)^{-}$ resonances are obvious, while no clear hint for $P_{c s}^{0}$ in the Dalitz plot Figure 3.

The amplitude model is constructed using helicity formalism, following a similar strategy in amplitude analysis of $\Lambda_{b}^{0} \rightarrow J / \psi p K^{-}$decays [6]. Two interfering decay chains are considered in the amplitude model: $\Xi_{b}^{-} \rightarrow J / \psi \Xi^{*-}\left(\rightarrow \Lambda K^{-}\right)$and $\Xi_{b}^{-} \rightarrow P_{c s}^{0}(\rightarrow J / \psi \Lambda) K^{-}$, as $J / \psi$ is reconstructed by $\mu^{+} \mu^{-}$pair and $\Lambda$ is reconstructed by proton and $\pi^{-}$.

The default amplitude model includes four excited $\Xi^{*-}$ resonances, $\Xi(1690)^{-}, \Xi(1820)^{-}$, $\Xi(1950)^{-}$and $\Xi(2030)^{-}, \operatorname{NR}\left(\Lambda K^{-}\right)$contribution with spin-parity quantum number equal to $J^{P}=$ $1 / 2^{-}$, and a single $P_{c s}^{0}$ contribution. The default model can describe data well, as shown in Figure 4. A statistical significance of $4.3 \sigma$ for $P_{c s}(4459)^{0}$ is obtained, while $3.1 \sigma$ including systematic uncertainties and look-elsewhere effect. This is the first evidence for the hidden-charm pentaquark with strangeness. The mass and width of this new pentaquark candidate $P_{c s}(4459)^{0}$ are measured to be $M_{P_{c s}}=4458.8 \pm 2.9_{-1.1}^{+4.7} \mathrm{MeV}$ and $\Gamma_{P_{c s}}=17.3 \pm 6.5_{-5.7}^{+8.0} \mathrm{MeV}$, respectively. Two $\Xi^{*-}$ states, $\Xi(1690)^{-}$and $\Xi(1820)^{-}$, are observed for the first time in $\Xi_{b}^{-}$decays. Due to the limited sample size, the spin-parity quantum number of $P_{c s}^{0}$ and $\Xi^{*-}$ resonances can not be determined. 

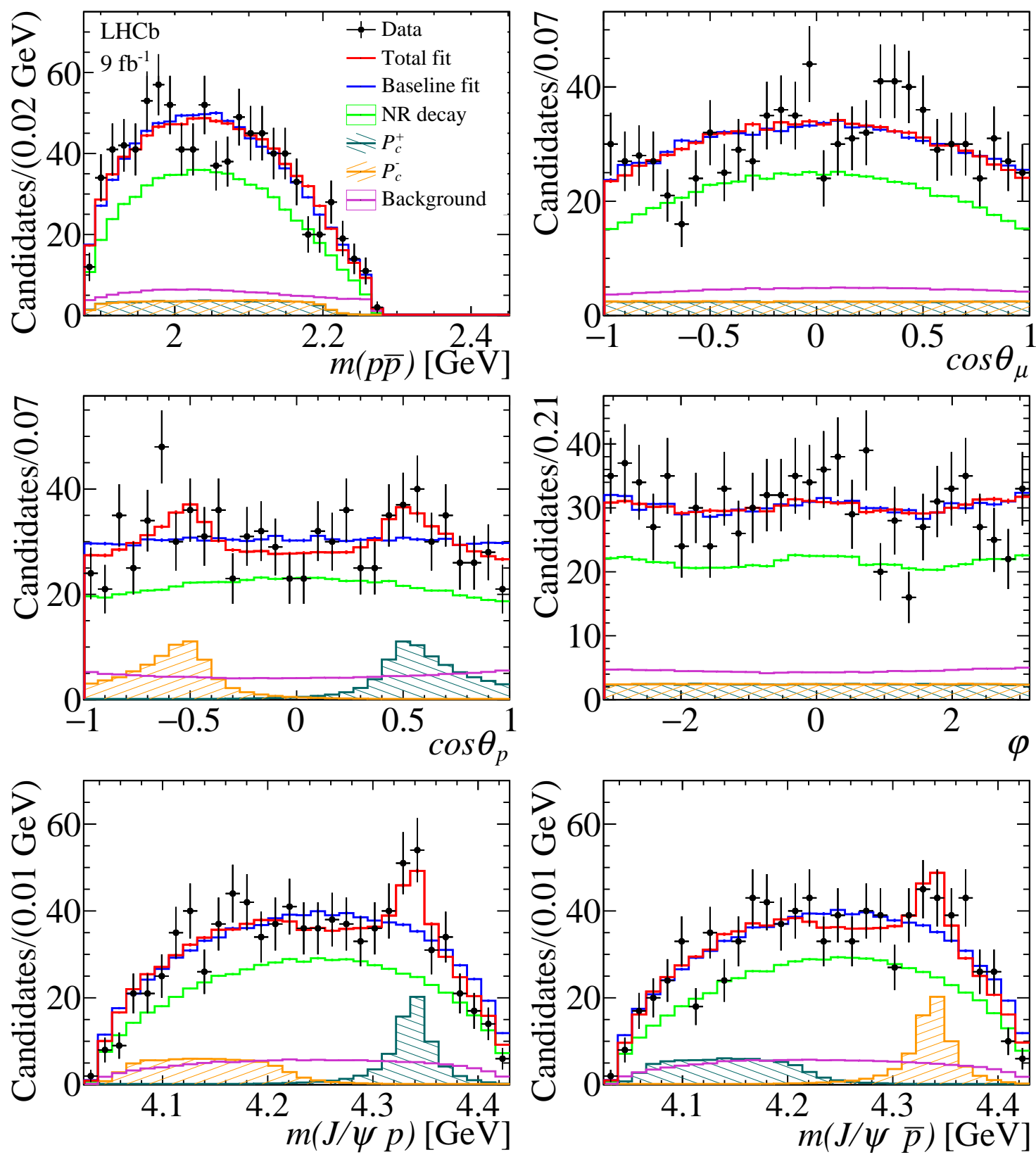

Figure 2: One-dimensional projections of the angular $\left(\cos \theta_{\mu}, \cos \theta_{p}, \varphi\right)$ and invariant-mass distributions $(m(p \bar{p}), m(J / \psi p), m(J / \psi \bar{p}))$, superimposed with the results of the fit from the baseline model (blue) and the default model (red) comprising a NR term and the $P_{c}$ contribution.

\section{Summary}

In summary, there are evidences for two new pentaquark candidates $P_{c}(4337)^{+}$and $P_{c s}(4459)^{0}$ in $B_{s}^{0} \rightarrow J / \psi p \bar{p}$ and $\Xi_{b}^{-} \rightarrow J / \psi \Lambda K^{-}$decays, respectively. The $P_{c}(4312)^{+}$observed in [1] is not confirmed, and no evidence for possible glueball candidate $f_{J}(2220)$ and enhancement in $p \bar{p}$ system from amplitude analysis of $B_{s}^{0} \rightarrow J / \psi p \bar{p}$ decays. 


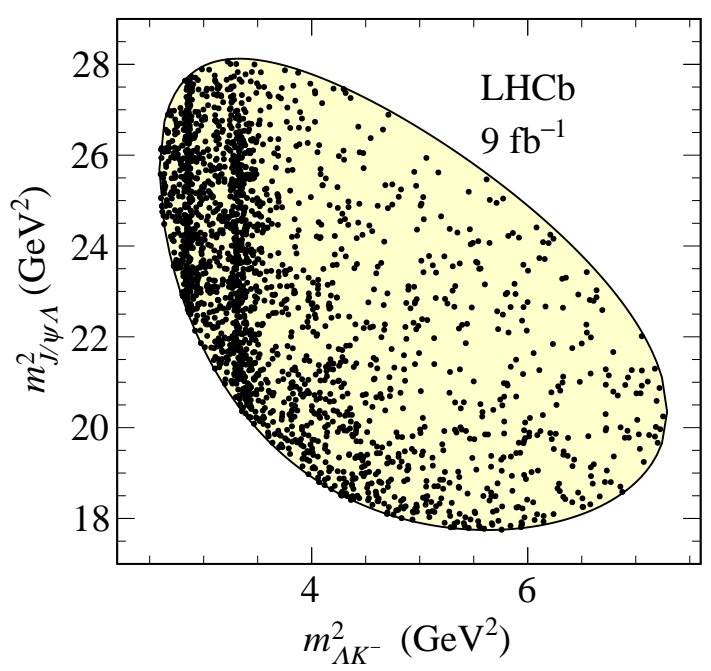

Figure 3: Dalitz plot for all candidates within $\pm 15 \mathrm{MeV}$ of the known $\Xi_{b}^{-}$mass. The yellow area shows the kinematically allowed region.
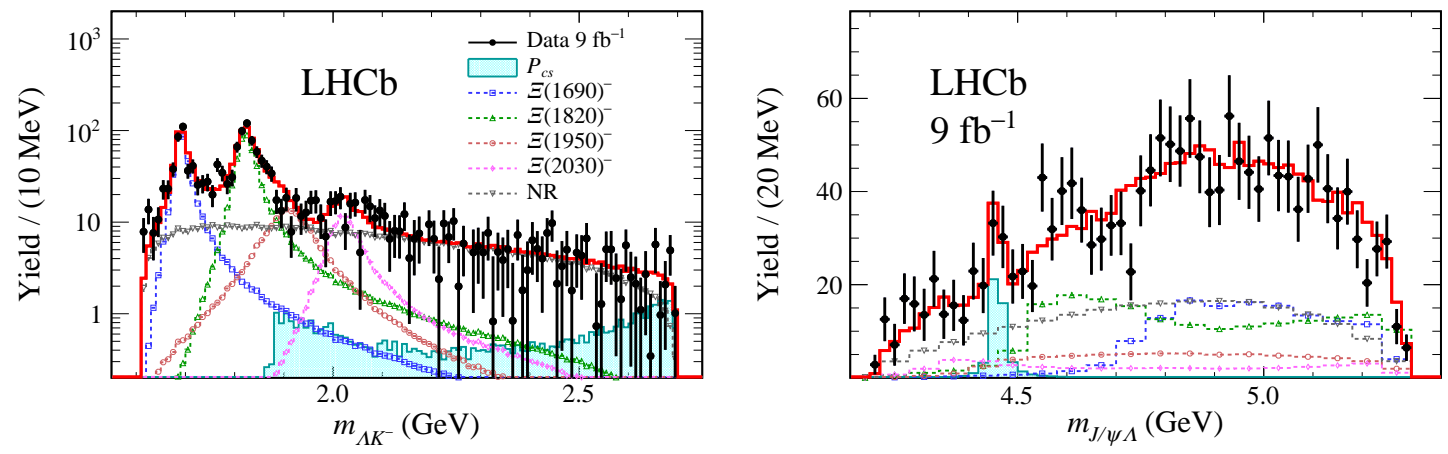

Figure 4: One-dimensional projections of mass distributions (a) $m_{\Lambda K^{-}}$and (b) $m_{J / \psi \Lambda}$ superimposed with the results of the fit with the $P_{c s}^{0}$ contribution. 


\section{References}

[1] LHCb collaboration, R. Aaij et al., Observation of a narrow pentaquark state, $P_{c}(4312)^{+}$, and of two-peak structure of the $P_{c}(4450)^{+}$, Phys. Rev. Lett. 122 (2019) 222001, [arXiv: 1904.03947].

[2] LHCb collaboration, R. Aaij et al., Observation of $B_{(s)}^{0} \rightarrow J / \psi p \bar{p}$ decays and precision measurements of the $B_{(s)}^{0}$ masses, Phys. Rev. Lett. 122 (2019) 191804, [arXiv: 1902 . 0558].

[3] LHCb collaboration, R. Aaij et al., Evidence for a new structure in the $J / \psi p$ and $J / \psi \bar{p}$ systems in $B_{s}^{0} \rightarrow J / \psi p \bar{p}$ decays, [arXiv:2108.04720].

[4] LHCb collaboration, R. Aaij et al., Observation of the $\Xi_{b}^{-} \rightarrow J / \psi \Lambda K^{-}$decay, Phys. Lett. $B$ 772 (2017) 265, [arXiv: 1701.05274].

[5] LHCb collaboration, R. Aaij et al., Evidence of a $J / \psi \Lambda$ structure and observation of excited $\Xi^{-}$states in the $\Xi_{b}^{-} \rightarrow J / \psi \Lambda K^{-}$decay, Sci. Bull. 66 (2021) 1278, [arXiv: 2012 . 10380].

[6] LHCb collaboration, R. Aaij et al., Observation of $J / \psi p$ Resonances Consistent with Pentaquark States in $\Lambda_{b}^{0} \rightarrow J / \psi K^{-} p$ Decays, Phys. Rev. Lett. 115 (2015) 072001, [arXiv: 1507.03414]. 